\title{
Phase noise-robust channel estimation for simple ICI compensation of millimeter wave OFDM systems
}

\author{
Yuyuan Changa), Gia Khanh Tran, Kei Sakaguchi, \\ and Kiyomichi Araki \\ Tokyo Institute of Technology, Tokyo, 152-8550 Japan \\ a) chang.y.ab@m.titech.ac.jp
}

\begin{abstract}
Although $60 \mathrm{GHz}$ millimeter wave (mm-Wave) systems employing OFDM can provide multi-Gbps data rate, the high level of phase noise (PN) of the mm-Wave transceivers induces inter-carrier interference (ICI) that degrades the performance of the OFDM systems. In the conventional method, the effects of PN can be well compensated by the iterative receiver processing; however, it has high complexity. In the previous work, we proposed a simple frequency domain ICI compensation, that linearly combines the channel equalized receive signals; however, it needs PN-robust channel estimation methods. In this paper we evaluate the proposed scheme with considering the channel estimation, and propose a PN-robust channel estimation method.
\end{abstract}

Keywords: millimeter wave, phase noise, OFDM, inter-carrier interference, compensation, channel estimation

Classification: Wireless Communication Technologies

\section{References}

[1] IEEE Std. 802.11ad-2012. IEEE SA., 24 Dec. 2012. DOI:10.1109/IEEESTD. 2012.6392842

[2] S. Suyama, H. Suzuki, K. Fukawa, and J. Izumi, "Iterative receiver employing phase noise compensation and channel estimation for millimeter-wave OFDM systems," IEEE J. Sel. Areas Commun., vol. 27, no. 8, pp. 1358-1366, Oct. 2009. DOI:10.1109/JSAC.2009.091006

[3] Y. Chang et al., "Simple inter-carrier interference compensation scheme for millimeter wave OFDM systems: PER evaluation considering channel estimation," IEICE Tech. Report, vol. 115, no. 473, pp. 151-156, Mar. 2016.

[4] P. Smulders, "Statistical characterization of $60-\mathrm{GHz}$ indoor radio channels," IEEE Trans. Antennas Propag., vol. 57, no. 10, pp. 2820-2829, Oct. 2009. DOI:10.1109/TAP.2009.2030524

[5] S. Coleri, M. Ergen, A. Puri, and A. Bahai, "Channel estimation techniques based on pilot arrangement in OFDM systems," IEEE Trans. Broadcast., vol. 48, no. 3, pp. 223-229, Sep. 2002. DOI:10.1109/TBC.2002.804034

[6] Y. Chang, K. Hirota, G. K. Tran, K. Sakaguchi, and K. Araki, “A study of user scheduling for millimeter wave MU-MIMO systems: evaluation with small 
conference room channel model and 3-sector access point," IEICE Tech. Report, vol. 115 , no. 288 , pp. 181-186, Nov. 2015.

\section{Introduction}

Millimeter wave (mm-Wave) wireless communications with OFDM are widely evaluated and adopted in the $60 \mathrm{GHz}$-based mm-Wave standards like IEEE 802.11ad [1]. However, the high level phase noise (PN) of the mm-Wave transceiver induces the common phase error (CPE) and inter-carrier interference (ICI) that degrade the performance of the OFDM systems. In the conventional method [2], the effects of PN are well compensated by the iterative receiver processing; however, it has very high complexity. In [3], we proposed a simple frequency domain scheme, which is expected to have much lower numerical complexity. However, PN-robust channel estimations are necessary. Here we evaluate the channel estimation methods, and propose a PN-robust channel estimation method for the proposed scheme.

\section{Simple frequency domain $\mathrm{ICl}$ compensation}

We consider a mm-Wave wireless link with one single antenna transmitter (Tx) and one single antenna receiver $(\mathrm{Rx})$. The Tx signal is defined as

$$
x(n)=\frac{\exp \left[\mathrm{j} \phi_{\mathrm{t}}(n)\right]}{\sqrt{N}} \sum_{m^{\prime}=0}^{N-1} X\left(m^{\prime}\right) \exp \left[\mathrm{j} 2 \pi \frac{m^{\prime}\left(n-N_{\mathrm{GI}}\right)}{N}\right],
$$

where $n$ is the index of time samples sampled with sampling rate $f_{\mathrm{s}} \cdot m^{\prime}$ is the index of the OFDM subcarriers, $N$ is the number of FFT points, $X\left(m^{\prime}\right)$ is the signal at the $m^{\prime}$ th subcarrier in frequency domain and $N_{\mathrm{GI}}$ is the length of guard interval (GI). $\phi_{\mathrm{t}}(n)$ is the PN in the Tx. Then the Rx signal is

$$
y(n)=\exp \left[\mathrm{j} \phi_{\mathrm{r}}(n)\right]\left[\sum_{d=0}^{D} h_{d} x(n-d)+v(n)\right],
$$

where $\phi_{\mathrm{r}}(n)$ is the Rx PN, $h_{d}$ is the $d$ th tap of the channel impulse response between the Tx and $\mathrm{Rx}$ antennas with delay $d$, and $v(n)$ is the received additive white Gaussian noise (AWGN) with variance $\sigma_{v}^{2}$ and zero mean. $D$ is the maximum delay of the channel and is assumed $D<N_{\mathrm{GI}}$.

After GI removal and FFT, the Rx signal at the $m$ th subcarrier becomes

$$
Y(m)=\frac{1}{\sqrt{N}} \sum_{n^{\prime}=0}^{N-1} y\left(n^{\prime}+N_{\mathrm{GI}}\right) \exp \left(-\mathrm{j} 2 \pi \frac{m n^{\prime}}{N}\right) .
$$

Substitute (1) and (2) into (3), then the received signal becomes

$$
\begin{aligned}
Y(m)= & \sum_{m^{\prime}=0}^{N-1} X\left(m^{\prime}\right) \sum_{d=0}^{D} h_{d} \Phi\left(d, m^{\prime}-m\right) \exp \left(-\mathrm{j} 2 \pi \frac{m^{\prime} d}{N}\right)+V^{\prime}(m) \\
\approx & \tilde{\Phi}(0) H(m) X(m)+\tilde{\Phi}(-1) H(m-1) X(m-1) \\
& +\tilde{\Phi}(1) H(m+1) X(m+1)+\sum_{\substack{m^{\prime}=0, m^{\prime} \notin[m-1, m+1]}}^{N-1} H^{\prime}\left(m^{\prime}, \Delta m\right) X\left(m^{\prime}\right)+V^{\prime}(m),
\end{aligned}
$$


In (4), $V^{\prime}(m)$ is the FFT converted $v(n)$ with the Rx PN, and $\Phi\left(d, m^{\prime}-m\right)$ is the FFT converted result of the PN with delay $d$ that becomes

$$
\Phi\left(d, m^{\prime}-m\right)=\frac{1}{N} \sum_{n^{\prime}=0}^{N-1} \exp \left[\mathrm{j} \hat{\phi}\left(n^{\prime}, d\right)\right] \exp \left[\mathrm{j} 2 \pi \frac{\left(m^{\prime}-m\right) n^{\prime}}{N}\right],
$$

where $\hat{\phi}\left(n^{\prime}, d\right)=\phi_{\mathrm{r}}\left(n^{\prime}+N_{\mathrm{GI}}\right)+\phi_{\mathrm{t}}\left(n^{\prime}+N_{\mathrm{GI}}-d\right)$. The transfer function (TF) of the channel then becomes

$$
H(m)=\sum_{d=0}^{D} h_{d} \exp \left(-\mathrm{j} 2 \pi \frac{m d}{N}\right)
$$

and the 2-dimensional TF, $H^{\prime}\left(m^{\prime}, \Delta m\right)$, including the $\mathrm{PN}$ is defined as

$$
H^{\prime}\left(m^{\prime}, \Delta m\right)=\sum_{d=0}^{D} h_{d} \Phi(d, \Delta m) \exp \left(-\mathrm{j} 2 \pi \frac{m^{\prime} d}{N}\right) .
$$

The approximation in (4) holds, when $\Phi\left(d, m^{\prime}-m\right) \approx \Phi\left(0, m^{\prime}-m\right) \triangleq \tilde{\Phi}(\Delta m)$, $\forall m^{\prime} \in[m-1, m+1]$ and $\Delta m \triangleq m^{\prime}-m$. The first term of (4) after the approximation is the desired signal multiplied with the CPE and the TF; the second and third terms are the ICI from the adjacent subcarriers; and the summation term is the remained ICI from the other subcarriers. $\tilde{\Phi}(0)$ is the coefficient of CPE, and $\tilde{\Phi}(\Delta m)$, $\forall \Delta m \neq 0$, is the coefficient of ICI from the $\Delta m$ th adjacent subcarrier. After channel equalization, (4) becomes

$$
\begin{aligned}
\tilde{X}(m) \triangleq & \frac{Y(m)}{\hat{H}(m)} \approx \tilde{\Phi}(0) X(m)+\tilde{\Phi}(-1) X(m-1)+\tilde{\Phi}(1) X(m+1) \\
& +\sum_{m^{\prime}=0, m^{\prime} \notin[m-1, m+1]}^{N-1} \frac{H^{\prime}\left(m^{\prime}, \Delta m\right)}{\hat{H}(m)} X\left(m^{\prime}\right)+\frac{V^{\prime}(m)}{\hat{H}(m)},
\end{aligned}
$$

where $\hat{H}(m)$ is the estimated TF that will be discussed in the following section, and the second approximation holds when $\hat{H}(m) \approx H(m)$ and $H(m+1) \approx H(m) \approx$ $H(m-1)$. The former is depending on the accuracy of channel estimation, and the latter implies that the TFs of the adjacent subcarriers should be similar to each other. In [3], the adaptive 3-tap frequency domain equalizer is adopted to compensate the CPE and cancel the adjacent ICIs. The same scheme is employed as shown below for $\tilde{X}(m)$ in (8).

$$
\hat{X}(m)=\mathbf{w}^{H} \tilde{\mathbf{x}}(m),
$$

where $\tilde{\mathbf{x}}(m)=[\tilde{X}(m-1) \tilde{X}(m) \tilde{X}(m+1)]^{T} ;$ and $\mathbf{w}=\left[W_{-1} W_{0} W_{1}\right]^{T}$ is the vector of CPE/ICI compensation weights that can be obtained as

$$
\mathbf{w}^{(\mathrm{opt})}=\left[\tilde{\mathbf{X}}^{(\mathrm{p})} \tilde{\mathbf{X}}^{(\mathrm{p}) H}\right]^{-1} \tilde{\mathbf{X}}^{(\mathrm{p})} \mathbf{x}^{(\mathrm{p}) *},
$$

using the pilot subcarriers in each OFDM symbol. In the equation, $\mathbf{x}^{(\mathrm{p})} \in \mathbb{C}^{N_{\mathrm{p}} \times 1}$ is the vector of transmitted pilots, where $N_{\mathrm{p}}$ is the number of pilot subcarriers; and $\tilde{\mathbf{X}}^{(\mathrm{p})} \in \mathbb{C}^{3 \times N_{\mathrm{p}}}$ includes the vectors of $\tilde{\mathbf{x}}(m)$ corresponding to the transmitted pilot subcarriers. A Tx packet includes two preambles at the head of packet and several data symbols following the preambles [3]. The first and second preambles are for the timing recovery and channel estimation, respectively. Each data symbol has several pilot subcarriers for the estimation of CPE/ICI compensation weights. Note that, the construction of channel estimation preamble will be further discussed in the following section. It is expected to reduce almost half of the ICI power with the 
proposed scheme, for the ratio of the adjacent ICI power to total ICI power is about $47 \%$ [3].

\section{Phase noise-robust channel estimation}

In [5], several channel estimation schemes were compared for OFDM systems, which can be divided into two types: block-type and comb-type. In block-type, the pilots are inserted into all of the subcarriers of the channel estimation preamble. On the other hand, in comb-type, the pilots are inserted into the pilot subcarriers, which are separated by several data subcarriers in each OFDM symbol. In this paper, we compare these two types of channel estimation schemes that are employed in the channel estimation preamble. Especially, in the comb-type preamble, we insert one null data into each subcarrier between two pilot subcarriers, i.e., the pilots are inserted at one subcarrier interval. It is expected to reduce almost half of ICI (for the reason discussed in [3]), and keep the estimation accuracy with the single subcarrier interval. The comb-type channel estimation consists of channel estimation at pilot subcarriers and interpolation for the TF. In [5], the interpolation methods include: linear interpolation (LI), second order interpolation (SOI), and time domain interpolation (TDI). TDI is a high-resolution method based on DFT/IDFT and zero-padding. After obtaining the estimated channel at each pilot in the preamble, $H^{(\mathrm{p})}\left(m_{\mathrm{p}}\right), m_{\mathrm{p}}=0,1, \ldots, N_{\mathrm{p}}^{\prime}-1$, it is first converted to the time domain by IDFT:

$$
h^{(\mathrm{p})}\left(n_{\mathrm{p}}\right)=\frac{1}{N_{\mathrm{p}}^{\prime}} \sum_{m_{\mathrm{p}}=0}^{N_{\mathrm{p}}^{\prime}-1} H^{(\mathrm{p})}\left(m_{\mathrm{p}}\right) \exp \left(\mathrm{j} 2 \pi \frac{m_{\mathrm{p}} n_{\mathrm{p}}}{N_{\mathrm{p}}^{\prime}}\right) .
$$

where $N_{\mathrm{p}}^{\prime}$ is the number of pilots in the preamble, $H^{(\mathrm{p})}\left(m_{\mathrm{p}}\right)=Y^{\prime}\left(2 m_{\mathrm{p}}+1\right) /$ $X^{\prime(\mathrm{p})}\left(m_{\mathrm{p}}\right), m_{\mathrm{p}}$ is the index of the pilots, $X^{\prime(\mathrm{p})}$ is the pilot signal in the preamble, and $Y^{\prime}$ is the frequency signal of the received preamble. Note that we select the odd subcarriers as pilots to avoid the DC offset at the 0th subcarrier. Then, the signal is interpolated by transforming the $N_{\mathrm{p}}^{\prime}$ points into $2 N_{\mathrm{p}}^{\prime}$ points with the basic multi-rate signal processing properties:

$$
\tilde{h}\left(n_{\mathrm{p}}\right)= \begin{cases}h^{(\mathrm{p})}\left(n_{\mathrm{p}}\right), & 0 \leq n_{\mathrm{p}}<N_{\mathrm{p}}^{\prime} / 2 \\ h^{(\mathrm{p})}\left(n_{\mathrm{p}}-N_{\mathrm{p}}^{\prime}\right), & 3 N_{\mathrm{p}}^{\prime} / 2 \leq n_{\mathrm{p}}<2 N_{\mathrm{p}}^{\prime} \\ 0, & \text { otherwise }\end{cases}
$$

where $n_{\mathrm{p}}=0,1, \ldots, 2 N_{\mathrm{p}}^{\prime}-1$, is the index of the time-domain interpolated impulse response $\tilde{h}$. It is converted into the frequency domain by DFT:

$$
\tilde{H}\left(m_{\mathrm{p}}^{\prime}\right)=\sum_{n_{\mathrm{p}}=0}^{2 N_{\mathrm{p}}^{\prime}-1} \tilde{h}\left(n_{\mathrm{p}}\right) \exp \left[-\mathrm{j} 2 \pi \frac{\left(m_{\mathrm{p}}^{\prime}-1\right) n_{\mathrm{p}}}{2 N_{\mathrm{p}}^{\prime}}\right],
$$

where $m_{\mathrm{p}}^{\prime}=0,1, \ldots, 2 N_{\mathrm{p}}^{\prime}-1$, and the -1 in $\left(m_{\mathrm{p}}^{\prime}-1\right)$ is for we select the odd subcarriers as the pilots. The estimated TF of all the subcarriers become:

$$
\hat{H}(m)= \begin{cases}\tilde{H}(m), & 0 \leq m<N_{\mathrm{p}}^{\prime} \\ \tilde{H}\left(m-N_{\mathrm{p}}^{\prime}\right), & N-N_{\mathrm{p}}^{\prime} \leq m<N \\ 0, & \text { otherwise }\end{cases}
$$

We conduct a simulation to compare the estimation accuracy of the schemes with the simulation parameters shown in the next section. We compare the mean 


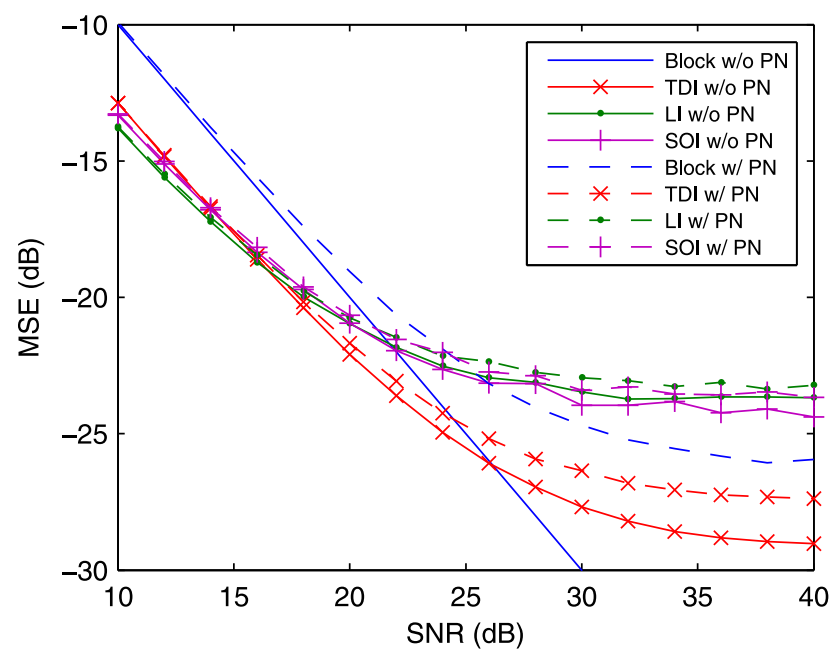

Fig. 1. MSE of different channel estimation schemes.

squared error (MSE) performances of the channel estimation methods; and the simulation results are shown in Fig. 1. Note that the number of pilots of the combtype is half of the block-type, so it has $3 \mathrm{~dB}$ SNR gain for each comb-type pilot. Without PN, when SNR is higher than $26 \mathrm{~dB}$, the block-type scheme has lower MSE level. The error floors of the comb-type schemes appear due to the interpolation accuracy of the schemes. With PN, the performance of the block-type scheme degrades significantly, when SNR is larger than $25 \mathrm{~dB}$, and the comb-type scheme with TDI has the best performance in the channel estimation schemes; and thus the comb-type scheme with TDI is adopted in the following performance evaluation as the PN-robust channel estimation. An important point is that, the estimation error due to the $\mathrm{CPE}$ is not counted in the MSE; for CPE of the estimated TF can be easily compensated in the following data symbols together with the CPE of the individual OFDM symbols.

\section{Computer simulation}

The simulation is conducted to evaluate the proposed scheme (FD-PNC), with the parameters shown in Table I. It is compared with the conventional schemes: CPE compensation only, (CPEC), and iterative PN compensation scheme (DD-PNC) [2]. Fig. 2 shows the average packet error rates (PER) of the schemes. From the results, the performance of "DD-PNC TDI" is the nearest to system without PN but with prefect TF (w/o PN ideal $\mathrm{Ch}$ ), while "CPEC ideal Ch" degrades significantly even when SNR is large. At PER $=10^{-2}$, the SNR degradations from "DD-PNC TDI" of "FD-PNC ideal Ch" and "FD-PNC TDI" are $2.6 \mathrm{~dB}$ and $5.0 \mathrm{~dB}$, respectively; however, the PER of "FD-PNC TDI" is still one order of magnitude smaller then "CPEC ideal Ch", when SNR is $30 \mathrm{~dB}$. We count the the numbers of real multiplications as the complexities for the proposed simple frequency domain scheme and the conventional iterative method. In the proposed scheme, the complexities of (9) and (10) are $\left(12 N_{\mathrm{d}}\right)$ and $\left(84 N_{\mathrm{p}}+144\right)$, respectively, where $N_{\mathrm{d}}$ is the number of data subcarriers. For channel estimation, the complexities of (11) and (13) are $\left(4 N_{\mathrm{p}}^{\prime 2}\right)$ and $\left(8 N_{\mathrm{p}}^{\prime 2}\right)$, respectively. Note that the channel estimation is conducted once per packet, so with the simulation parameters, the complexity of the proposed 
Table I. Simulation parameters.

\begin{tabular}{|c|c|}
\hline Parameter & Value or Setting \\
\hline Sampling rate & $2.640 \mathrm{GHz}$ \\
\hline Transmission scheme & OFDM (64QAM) \\
\hline No. of FFT/GI points & $512 / 128$ \\
\hline Packet construction & Preamble: 2 , data symbol: 12 \\
\hline No. of pilots in preamble & Block: 360, comb: 180 \\
\hline $\begin{array}{l}\text { No. of active subcarriers } \\
\text { in data symbols }\end{array}$ & Data: 336 , pilot: 16 \\
\hline Channel coding & LDPC $(672,546)$ \\
\hline Channel decoding & $\begin{array}{l}\text { Sum-product algorithm } \\
\text { (max. } 8 \text { iterations) }\end{array}$ \\
\hline Data rate & $6.76 \mathrm{Gbps}$ \\
\hline DDCE \& DD-PNC [2] & \\
\hline Max. no. of iterations & 5 (except the initial process) \\
\hline Step-size: $\mu_{c}, \mu_{\phi}$ & $0.001,0.01-0.1$ \\
\hline Channel model [6] & $\begin{array}{c}\text { Nakagami-Rician }(K=10 \mathrm{~dB}) \\
1 \text { LOS }+5 \text { NLOS clusters } \\
(6 \text { components } / \text { cluster })\end{array}$ \\
\hline Channel estimation & Ideal, or comb-type with TDI \\
\hline Total PN level of Tx and Rx & $\begin{array}{l}-88 \mathrm{dBc} / \mathrm{Hz} @ 1 \mathrm{MHz} \text { offset [2]. } \\
\text { Generated by AR model. }\end{array}$ \\
\hline
\end{tabular}

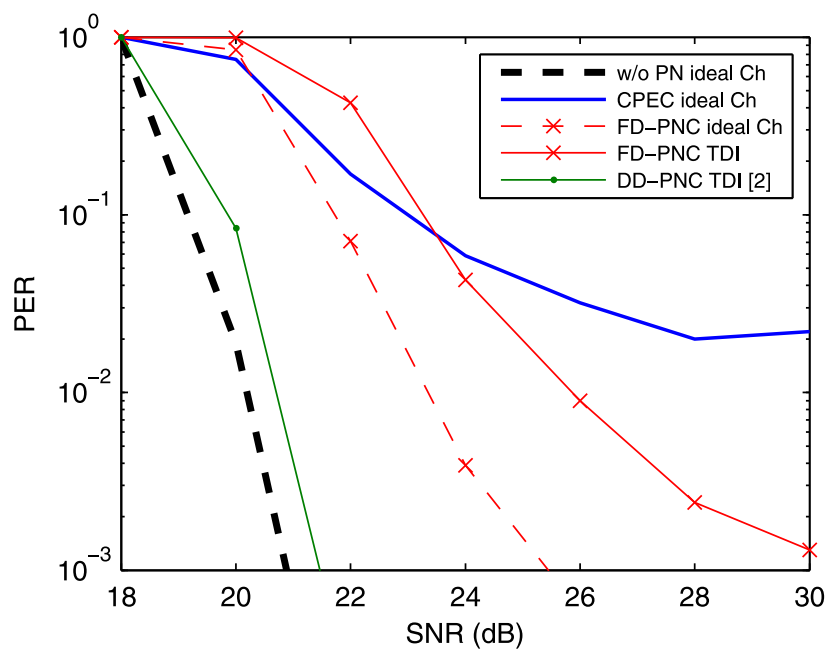

Fig. 2. Average PER of different schemes.

scheme is $5.5 \times 10^{3}$ per symbol beside the channel estimation. On the other hand, in [2], for each symbol, the complexities of DD-PNC and DDCE are $(22 N+$ $\left.4 N \log _{2} N\right)$ and $\left(10 \Omega N+4 N \log _{2} N\right)$, respectively, where $\Omega$ is the number of extracted paths after the path detection (selection). With the simulation parameters and setting $\Omega=32$, the complexity of the conventional scheme becomes $2.1 \times 10^{5}$ per symbol. Obviously, the proposed scheme has much lower complexity. 


\section{Conclusion}

We proposed a simple frequency domain ICI compensation scheme with phase noise-robust channel estimation. Although, comparing with the conventional iterative scheme, the proposed scheme has several dB SNR degradation, it has much lower numerical complexity, and is expected to have lower and fixed latency that let it can be easily implemented.

\section{Acknowledgments}

This work was partly supported by "The research and development project for expansion of radio spectrum resources" of The Ministry of Internal Affairs and Communications, Japan. 